\title{
Iterative Solution to a System of Matrix Equations
}

\author{
Yong Lin ${ }^{1,2}$ and Qing-Wen Wang ${ }^{1}$ \\ ${ }^{1}$ Department of Mathematics, Shanghai University, Shanghai 200444, China \\ ${ }^{2}$ School of Mathematics and Statistics, Suzhou University, Suzhou 234000, China \\ Correspondence should be addressed to Qing-Wen Wang; wqw369@yahoo.com
}

Received 17 May 2013; Accepted 21 September 2013

Academic Editor: Masoud Hajarian

Copyright (C) 2013 Y. Lin and Q.-W. Wang. This is an open access article distributed under the Creative Commons Attribution License, which permits unrestricted use, distribution, and reproduction in any medium, provided the original work is properly cited.

An efficient iterative algorithm is presented to solve a system of linear matrix equations $A_{1} X_{1} B_{1}+A_{2} X_{2} B_{2}=E, C_{1} X_{1} D_{1}+$ $C_{2} X_{2} D_{2}=F$ with real matrices $X_{1}$ and $X_{2}$. By this iterative algorithm, the solvability of the system can be determined automatically. When the system is consistent, for any initial matrices $X_{1}^{0}$ and $X_{2}^{0}$, a solution can be obtained in the absence of roundoff errors, and the least norm solution can be obtained by choosing a special kind of initial matrix. In addition, the unique optimal approximation solutions $\widehat{X}_{1}$ and $\widehat{X}_{2}$ to the given matrices $\widetilde{X}_{1}$ and $\widetilde{X}_{2}$ in Frobenius norm can be obtained by finding the least norm solution of a new pair of matrix equations $A_{1} \bar{X}_{1} B_{1}+A_{2} \bar{X}_{2} B_{2}=\bar{E}, C_{1} \bar{X}_{1} D_{1}+C_{2} \bar{X}_{2} D_{2}=\bar{F}$, where $\bar{E}=E-A_{1} \widetilde{X}_{1} B_{1}-A_{2} \widetilde{X}_{2} B_{2}$, $\bar{F}=F-C_{1} \widetilde{X}_{1} D_{1}-C_{2} \widetilde{X}_{2} D_{2}$. The given numerical example demonstrates that the iterative algorithm is efficient. Especially, when the numbers of the parameter matrices $A_{1}, A_{2}, B_{1}, B_{2}, C_{1}, C_{2}, D_{1}, D_{2}$ are large, our algorithm is efficient as well.

\section{Introduction}

Throughout the paper, we denote the set of all $m \times n$ real matrix by $R^{m \times n}$, the transpose matrix of $A$ by $A^{T}$, the identity matrix of order $n$ by $I_{n}$, the Kronecker product of $A$ and $B$ by $A \otimes B$, the $m n \times 1$ vector formed by the vertical concatenation of the respective columns of a matrix $A \in R^{m \times n}$ by $\operatorname{vec}(A)$, the trace of a matrix $A$ by $\operatorname{tr}(A)$, and the Frobenius norm of a matrix $A$ by $\|A\|$ where $\|A\|=\sqrt{\operatorname{tr}\left(A^{T} A\right)}$.

In this paper, we consider the following two problems.

Problem 1. For the given matrices $A_{1} \in R^{p \times k}, A_{2} \in R^{p \times m}$, $B_{1} \in R^{r \times q}, B_{2} \in R^{n \times q}, C_{1} \in R^{s \times k}, C_{2} \in R^{s \times m}, D_{1} \in R^{r \times t}, D_{2} \in$ $R^{n \times t}, E \in R^{p \times q}$, and $F \in R^{s \times t}$, find $X_{1} \in R^{k \times r}$ and $X_{2} \in R^{m \times n}$ such that

$$
\begin{aligned}
& A_{1} X_{1} B_{1}+A_{2} X_{2} B_{2}=E, \\
& C_{1} X_{1} D_{1}+C_{2} X_{2} D_{2}=F .
\end{aligned}
$$

Problem 2. When Problem 1 is consistent, let $S$ denote the solution set of the pair of matrix equation (1). For the given matrices $\widetilde{X}_{1} \in R^{k \times r}, \widetilde{X}_{2} \in R^{m \times n}$, find $\left\{\widehat{X}_{1}, \widehat{X}_{2}\right\} \in S$ such that

$$
\begin{aligned}
\| \widehat{X}_{1} & -\widetilde{X}_{1}\left\|^{2}+\right\| \widehat{X}_{2}-\widetilde{X}_{2} \|^{2} \\
& =\min _{\left\{X_{1}, X_{2}\right\} \in S}\left(\left\|X_{1}-\widetilde{X}_{1}\right\|^{2}+\left\|X_{2}-\widetilde{X}_{2}\right\|^{2}\right) .
\end{aligned}
$$

Problem 2 is to find the optimal approximation solutions to the given matrices $\widetilde{X}_{1}, \widetilde{X}_{2}$ in the solution set of Problem 1 . It occurs frequently in experiment design (see, for instance, [1]). In the recent years, the matrix optimal approximation problem has been studied extensively (e.g., [2-13]).

The research on solving matrix equation pair has been actively ongoing for the last 30 years or more. For instance, Mitra [14] gave conditions for the existence of a solution and a representation of the general common solution to $A X B=E, C X D=F$. Shinozaki and Sibuya [15] and van der Woude [16] discussed conditions for the existence of a common solution to $A X B=E, C X D=F$. Navarra et al. [5] derived sufficient and necessary conditions for the existence of a common solution to $A X B=E, C X D=F$. Yuan [13] obtained an analytical expression of the least-squares solutions of $A X B=E, C X D=F$ by using the generalized 
singular value decomposition (GSVD) of matrices. Dehghan and Hajarian [17] presented some examples to show a motivation for studying the general coupled matrix equations $\sum_{j=1}^{l} A_{i j} X_{j} B_{i j}=C_{i}, i=1,2, \ldots, l$, and [18] constructed an iterative algorithm to solve the general coupled matrix equations $\sum_{j=1}^{p} A_{i j} X_{j} B_{i j}=M_{i}, i=1,2, \ldots, p$. Wang $[19,20]$ gave the centrosymmetric solution to the system of quaternion matrix equations $A_{1} X=C_{1}, A_{3} X B_{3}=C_{3}$. Wang [21] also solved a system of matrix equations over arbitrary regular rings with identity.

Recently, some finite iterative algorithms have also been developed to solve matrix equations. Ding et al. [22, 23] and Xie et al. $[24,25]$ studied the iterative solutions of matrix equations $A X B=F$ and $A_{i} X B_{i}=F_{i}$ and generalized Sylvester matrix equations $A X B+C X D=F$ and $A X B+C X^{T} D=$ $F$. They presented a gradient based and a least-squares based iterative algorithms for the solution. Li et al. [26, 27] and Zhou et al. [28, 29] considered iterative method for some coupled linear matrix equations. Deng et al. [30] studied the consistent conditions and the general expressions about the Hermitian solutions of the matrix equations $(A X, X B)=$ $(C, D)$ and designed an iterative method for its Hermitian minimum norm solutions. $\mathrm{Li}$ and $\mathrm{Wu}$ [31] gave symmetric and skew-antisymmetric solutions to certain matrix equations $A_{1} X=C_{1}, X B_{3}=C_{3}$ over the real quaternion algebra $\mathrm{H}$. For more studies on iterative algorithms on coupled matrix equations, we refer to [3, 10-12, 17, 32-37]. Peng et al. [6] presented iterative methods to obtain the symmetric solutions of $A X B=E, C X D=F$. Sheng and Chen [8] presented a finite iterative method; when $A X B=E, C X D=$ $F$ is consistent. Liao and Lei [38] presented an analytical expression of the least-squares solution and an algorithm for $A X B=E, C X D=F$ with the minimum norm. Peng et al. [7] presented an algorithm for the least-squares reflexive solution. Dehghan and Hajarian [2] presented an iterative algorithm for solving a pair of matrix equations $A X B=E, C X D=$ $F$ over generalized centrosymmetric matrices. Cai and Chen [39] presented an iterative algorithm for the least-squares bisymmetric solutions of the matrix equations $A X B=E$, $C X D=F$. Yin and Huang [40] presented an iterative algorithm to solve the least squares generalized reflexive solutions of the matrix equations $A X B=E, C X D=F$.

However, to our knowledge, there has been little information on finding the solutions to the system (1) by iterative algorithm. In this paper, an efficient iterative algorithm is presented to solve the system (1) for any real matrices $X_{1}, X_{2}$. The suggested iterative algorithm, automatically determines the solvability of equations pair (1). When the pair of equations is consistent, then, for any initial matrices $X_{1}^{0}$ and $X_{2}^{0}$, the solution can be obtained in the absence of round errors, and the least norm solution can be obtained by choosing a special kind of initial matrix. In addition, the unique optimal approximation solutions $\widehat{X}_{1}$ and $\widehat{X}_{2}$ to the given matrices $\widetilde{X}_{1}$ and $\widetilde{X}_{2}$ in Frobenius norm can be obtained by finding the least norm solution of a new pair of matrix equations $A_{1} \bar{X}_{1} B_{1}+A_{2} \bar{X}_{2} B_{2}=\bar{E}, C_{1} \bar{X}_{1} D_{1}+C_{2} \bar{X}_{2} D_{2}=\bar{F}$, where $\bar{E}=E-A_{1} \widetilde{X}_{1} B_{1}-A_{2} \widetilde{X}_{2} B_{2}, \bar{F}=F-C_{1} \widetilde{X}_{1} D_{1}-C_{2} \widetilde{X}_{2} D_{2}$.
The given numerical examples demonstrate that our iterative algorithm is efficient. Especially, when the numbers of the parameter matrices $A_{1}, A_{2}, B_{1}, B_{2}, C_{1}, C_{2}, D_{1}, D_{2}$ are large, our algorithm is efficient as well while the algorithm of [32] is not convergent. That is, our algorithm has merits of good numerical stability and ease to program.

The rest of this paper is outlined as follows. In Section 2, we first propose an efficient iterative algorithm for solving Problem 1; then we give some properties of this iterative algorithm. We show that the algorithm can obtain a solution group (the least Frobenius norm solution group) for any (special) initial matrix group in the absence of roundoff errors. In Section 3, a numerical example is given to illustrate that our algorithm is quite efficient.

\section{Iterative Algorithm for Solving Problems 1 and 2}

In this section, we present the iterative algorithm for the consistence of the system (1).

Algorithm 3. (1) Input matrices $A_{1} \in R^{p \times k}, A_{2} \in R^{p \times m}, B_{1} \in$ $R^{r \times q}, B_{2} \in R^{n \times q}, C_{1} \in R^{s \times k}, C_{2} \in R^{s \times m}, D_{1} \in R^{r \times t}, D_{2} \in R^{n \times t}$, $E \in R^{p \times q}, F \in R^{s \times t}, X_{1}^{1} \in R^{k \times r}$, and $X_{2}^{1} \in R^{m \times n}$ (where $X_{1}^{1}, X_{2}^{1}$ are any initial matrices).

(2) Calculate

$$
\begin{gathered}
E^{1}=E, \\
F^{1}=F, \\
P_{1}^{1}=A_{1}^{T} E^{1} B_{1}^{T}+C_{1}^{T} F^{1} D_{1}^{T}, \\
P_{2}^{1}=A_{2}^{T} E^{1} B_{2}^{T}+C_{2}^{T} F^{1} D_{2}^{T}, \\
\beta^{1}=\left(\operatorname{tr}\left[\left(E^{1}\right)^{T}\left(A_{1} P_{1}^{1} B_{1}+A_{2} P_{2}^{1} B_{2}\right)\right]\right. \\
\left.+\operatorname{tr}\left[\left(F^{1}\right)^{T}\left(C_{1} P_{1}^{1} D_{1}+C_{2} P_{2}^{1} D_{2}\right)\right]\right) \\
\times\left(\left\|A_{1} P_{1}^{1} B_{1}+A_{2} P_{2}^{1} B_{2}\right\|^{2}\right. \\
\left.+\left\|C_{1} P_{1}^{1} D_{1}+C_{2} P_{2}^{1} D_{2}\right\|^{2}\right)^{-1}, \\
\Delta X_{1}^{1}=\beta^{1} P_{1}^{1}, \\
\Delta X_{2}^{1}=\beta^{1} P_{2}^{1}, \\
k=1 .
\end{gathered}
$$

(3) If $\Delta X=\operatorname{diag}\left(\Delta X_{1}^{k}, \Delta X_{2}^{k}\right)=0(k=1,2, \ldots)$, then stop. Otherwise,

$$
\begin{aligned}
& X_{1}^{k+1}=X_{1}^{k}+\Delta X_{1}^{k} \\
& X_{2}^{k+1}=X_{2}^{k}+\Delta X_{2}^{k}
\end{aligned}
$$


(4) Calculate

$$
\begin{gathered}
E^{k+1}=E^{k}-\left(A_{1} \Delta X_{1}^{k} B_{1}+A_{2} \Delta X_{2}^{k} B_{2}\right), \\
F^{k+1}=F^{k}-\left(C_{1} \Delta X_{1}^{k} D_{1}+C_{2} \Delta X_{2}^{k} D_{2}\right), \\
P_{1}^{k+1}=A_{1}^{T} E^{k+1} B_{1}^{T}+C_{1}^{T} F^{k+1} D_{1}^{T}, \\
P_{2}^{k+1}=A_{2}^{T} E^{k+1} B_{2}^{T}+C_{2}^{T} F^{k+1} D_{2}^{T}, \\
\beta^{k+1}=\left(\operatorname{tr}\left[\left(E^{k+1}\right)^{T}\left(A_{1} P_{1}^{k+1} B_{1}+A_{2} P_{2}^{k+1} B_{2}\right)\right]\right. \\
\left.+\operatorname{tr}\left[\left(F^{k+1}\right)^{T}\left(C_{1} P_{1}^{k+1} D_{1}+C_{2} P_{2}^{k+1} D_{2}\right)\right]\right) \\
\times\left(\left\|A_{1} P_{1}^{k+1} B_{1}+A_{2} P_{2}^{k+1} B_{2}\right\|^{2}\right. \\
\left.+\left\|C_{1} P_{1}^{k+1} D_{1}+C_{2} P_{2}^{k+1} D_{2}\right\|^{2}\right)^{-1}, \\
\Delta X_{1}^{k+1}=\beta^{k+1} P_{1}^{k+1}, \\
\Delta X_{2}^{k+1}=\beta^{k+1} P_{2}^{k+1}, \\
k=k+1 .
\end{gathered}
$$

Go to (3).

Lemma 4. In Algorithm 3, the choice of $\beta^{k}$ makes $\| \operatorname{diag}\left(E^{k+1}\right.$, $\left.F^{k+1}\right) \|$ reach a minimum and $\operatorname{diag}\left(E^{k+1}, F^{k+1}\right)$ and $\operatorname{diag}\left(A_{1} \Delta X_{1}^{k} B_{1}+A_{2} \Delta X_{2}^{k} B_{2}, C_{1} \Delta X_{1}^{k} D_{1}+C_{2} \Delta X_{2}^{k} D_{2}\right)$ orthogonal to each other.

Proof. From Algorithm 3, we have

$$
\begin{gathered}
\left\|\operatorname{diag}\left(E^{k+1}, F^{k+1}\right)\right\|^{2} \\
=\| \operatorname{diag}\left(E^{k}-\left(A_{1} \Delta X_{1}^{k} B_{1}+A_{2} \Delta X_{2}^{k} B_{2}\right),\right. \\
\left.F^{k}-\left(C_{1} \Delta X_{1}^{k} D_{1}+C_{2} \Delta X_{2}^{k} D_{2}\right)\right) \|^{2} \\
=\| \operatorname{diag}\left(E^{k}-\left(A_{1} \beta^{k} P_{1}^{k} B_{1}+A_{2} \beta^{k} P_{2}^{k} B_{2}\right),\right. \\
\left.F^{k}-\left(C_{1} \beta^{k} P_{1}^{k} D_{1}+C_{2} \beta^{k} P_{2}^{k} D_{2}\right)\right) \|^{2} \\
=\left\|E^{k}-\left(A_{1} \beta^{k} P_{1}^{k} B_{1}+A_{2} \beta^{k} P_{2}^{k} B_{2}\right)\right\|^{2} \\
+\left\|F^{k}-\left(C_{1} \beta^{k} P_{1}^{k} D_{1}+C_{2} \beta^{k} P_{2}^{k} D_{2}\right)\right\|^{2} \\
=\left\|E^{k}\right\|^{2}+\left\|F^{k}\right\|^{2} \\
-2\left[\operatorname{tr}\left(E^{k}, A_{1} P_{1}^{k} B_{1}+A_{2} P_{2}^{k} B_{2}\right)\right. \\
\left.\quad+\operatorname{tr}\left(F^{k}, C_{1} P_{1}^{k} D_{1}+C_{2} P_{2}^{k} D_{2}\right)\right] \beta^{k} \\
+\left[\left\|A_{1} P_{1}^{k} B_{1}+A_{2} P_{2}^{k} B_{2}\right\|^{2}\right. \\
\left.\quad+\left\|C_{1} P_{1}^{k} D_{1}+C_{2} P_{2}^{k} D_{2}\right\|^{2}\right]\left(\beta^{k}\right)^{2} .
\end{gathered}
$$

From the above, the condition of $\left\|\operatorname{diag}\left(E^{k+1}, F^{k+1}\right)\right\|$ reaching a minimum is

$$
\begin{aligned}
\beta^{k}=( & \operatorname{tr}\left(E^{k}, A_{1} P_{1}^{k} B_{1}+A_{2} P_{2}^{k} B_{2}\right) \\
& \left.+\operatorname{tr}\left(F^{k}, C_{1} P_{1}^{k} D_{1}+C_{2} P_{2}^{k} D_{2}\right)\right) \\
\times & \left(\left\|A_{1} P_{1}^{k} B_{1}+A_{2} P_{2}^{k} B_{2}\right\|^{2}\right. \\
& \left.+\left\|C_{1} P_{1}^{k} D_{1}+C_{2} P_{2}^{k} D_{2}\right\|^{2}\right)^{-1} .
\end{aligned}
$$

On the other hand, if the choice of $\beta^{k}$ makes $\operatorname{diag}\left(E^{k+1}\right.$, $\left.F^{k+1}\right)$ and $\operatorname{diag}\left(A_{1} \Delta X_{1}^{k} B_{1}+A_{2} \Delta X_{2}^{k} B_{2}, C_{1} \Delta X_{1}^{k} D_{1}+C_{2} \Delta X_{2}^{k} D_{2}\right)$ orthogonal to each other, that is, $\operatorname{tr}\left[\operatorname{diag}\left(E^{k+1}\right.\right.$, $\left.\left.F^{k+1}\right)^{T} \operatorname{diag}\left(A_{1} \Delta X_{1}^{k} B_{1}+A_{2} \Delta X_{2}^{k} B_{2}, C_{1} \Delta X_{1}^{k} D_{1}+C_{2} \Delta X_{2}^{k} D_{2}\right)\right]=$ 0 , we can have the same $\beta^{k}$ as (7).

Theorem 5. Algorithm 3 is bound to be convergent.

Proof. From Algorithm 3 and Lemma 4 we have

$$
\begin{gathered}
\operatorname{diag}\left(E^{k}, F^{k}\right)=\operatorname{diag}\left(E^{k+1}, F^{k+1}\right) \\
+\operatorname{diag}\left(A_{1} \Delta X_{1}^{k} B_{1}+A_{2} \Delta X_{2}^{k} B_{2},\right. \\
\left.C_{1} \Delta X_{1}^{k} D_{1}+C_{2} \Delta X_{2}^{k} D_{2}\right), \\
\Longleftrightarrow\left\|\operatorname{diag}\left(E^{k}, F^{k}\right)\right\|^{2} \\
=\| \operatorname{diag}\left(E^{k+1}, F^{k+1}\right)+\operatorname{diag}\left(A_{1} \Delta X_{1}^{k} B_{1}+A_{2} \Delta X_{2}^{k} B_{2},\right. \\
\left.=\left\|\operatorname{diag}\left(E^{k+1}, F^{k+1}\right)\right\|^{2} \quad C_{1} \Delta X_{1}^{k} D_{1}+C_{2} \Delta X_{2}^{k} D_{2}\right) \|^{2} \\
+\| \operatorname{diag}\left(A_{1} \Delta X_{1}^{k} B_{1}+A_{2} \Delta X_{2}^{k} B_{2},\right. \\
\left.C_{1} \Delta X_{1}^{k} D_{1}+C_{2} \Delta X_{2}^{k} D_{2}\right) \|^{2}
\end{gathered}
$$

such that

$$
\begin{aligned}
& \left\|\operatorname{diag}\left(E^{k+1}, F^{k+1}\right)\right\|^{2} \\
& =\left\|\operatorname{diag}\left(E^{k}, F^{k}\right)\right\|^{2} \\
& -\| \operatorname{diag}\left(A_{1} \Delta X_{1}^{k} B_{1}+A_{2} \Delta X_{2}^{k} B_{2},\right. \\
& \left.C_{1} \Delta X_{1}^{k} D_{1}+C_{2} \Delta X_{2}^{k} D_{2}\right) \|^{2} \\
& \leq\left\|\operatorname{diag}\left(E^{k}, F^{k}\right)\right\|^{2} .
\end{aligned}
$$

From (9), we know that Algorithm 3 is convergent.

Lemma 6 (see [41]). Suppose that the consistent system of linear equations $M y=b$ has a solution $y_{0} \in R\left(M^{T}\right)$; then $y_{0}$ is the least Frobenius norm solution of the system of linear equations. 
Theorem 7. Assume that the system (1) is consistent. Let $X_{1}^{1}=$ $A_{1}^{T} Y B_{1}^{T}+C_{1}^{T} Z D_{1}^{T}, X_{2}^{1}=A_{2}^{T} Y B_{2}^{T}+C_{2}^{T} Z D_{2}^{T}$ be initial matrices where $Y \in R^{p \times q}, Z \in R^{s \times t}$ are any initial matrices, or, especially, $X_{1}^{1}=0, X_{2}^{1}=0$; then the solution generated by Algorithm 3 is the least Frobenius norm solution to (1).

Proof. If (1) is consistent, from $X_{1}^{1}=A_{1}^{T} Y B_{1}^{T}+C_{1}^{T} Z D_{1}^{T}, X_{2}^{1}=$ $A_{2}^{T} Y B_{2}^{T}+C_{2}^{T} Z D_{2}^{T}$, using Algorithm 3, we have the iterative solution pair $X_{1}^{k}, X_{2}^{k}$ of (1) as the following:

$$
\begin{aligned}
X_{1}^{k}= & X_{1}^{k-1}+\Delta X_{1}^{k-1} \\
= & X_{1}^{1}+\Delta X_{1}^{1}+\cdots+\Delta X_{1}^{k-1} \\
= & A_{1}^{T} Y B_{1}^{T}+C_{1}^{T} Z D_{1}^{T} \\
& +A_{1}^{T}\left[\beta^{1} E^{1}+\cdots+\beta^{k-1} E^{k-1}\right] B_{1}^{T} \\
& +C_{1}^{T}\left[\beta^{1} F^{1}+\cdots+\beta^{k-1} F^{k-1}\right] D_{1}^{T} \\
= & A_{1}^{T} M B_{1}^{T}+C_{1}^{T} N D_{1}^{T}, \\
X_{2}^{k}= & X_{2}^{k-1}+\Delta X_{2}^{k-1} \\
= & X_{2}^{1}+\Delta X_{2}^{1}+\cdots+\Delta X_{1}^{k-1} \\
= & A_{2}^{T} Y B_{2}^{T}+C_{2}^{T} Z D_{2}^{T} \\
& +A_{2}^{T}\left[\beta^{1} E^{1}+\cdots+\beta^{k-1} E^{k-1}\right] B_{2}^{T} \\
& +C_{2}^{T}\left[\beta^{1} F^{1}+\cdots+\beta^{k-1} F^{k-1}\right] D_{2}^{T} \\
= & A_{2}^{T} M B_{2}^{T}+C_{2}^{T} N D_{2}^{T} .
\end{aligned}
$$

We know that (1) is equivalent to the system

$$
\left(\begin{array}{cc}
B_{1}^{T} \otimes A_{1} & B_{2}^{T} \otimes A_{2} \\
D_{1}^{T} \otimes C_{1} & D_{2}^{T} \otimes C_{2}
\end{array}\right)\left(\begin{array}{c}
\operatorname{vec}\left(X_{1}\right) \\
\operatorname{vec}\left(X_{2}\right)
\end{array}\right)=\left(\begin{array}{c}
\operatorname{vec}(E) \\
\operatorname{vec}(F)
\end{array}\right) .
$$

From (10) and (11) we have

$$
\begin{aligned}
& \left(\begin{array}{c}
\operatorname{vec}\left(A_{1}^{T} M B_{1}^{T}+C_{1}^{T} N D_{1}^{T}\right) \\
\operatorname{vec}\left(A_{2}^{T} M B_{2}^{T}+C_{2}^{T} N D_{2}^{T}\right)
\end{array}\right) \\
& =\left(\begin{array}{cc}
B_{1} \otimes A_{1}^{T} & D_{1} \otimes C_{1}^{T} \\
B_{2} \otimes A_{2}^{T} & D_{2} \otimes C_{2}^{T}
\end{array}\right)\left(\begin{array}{c}
\operatorname{vec}(M) \\
\operatorname{vec}(N)
\end{array}\right) \\
& =\left(\begin{array}{cc}
B_{1}^{T} \otimes A_{1} & B_{2}^{T} \otimes A_{2} \\
D_{1}^{T} \otimes C_{1} & D_{2}^{T} \otimes C_{2}
\end{array}\right)^{T} \\
& \times\left(\begin{array}{c}
\operatorname{vec}(M) \\
\operatorname{vec}(N)
\end{array}\right) \in R\left(\left(\begin{array}{cc}
B_{1}^{T} \otimes A_{1} & B_{2}^{T} \otimes A_{2} \\
D_{1}^{T} \otimes C_{1} & D_{2}^{T} \otimes C_{2}
\end{array}\right)^{T}\right),
\end{aligned}
$$

where $R(*)$ is the column space of matrix *.

Considering Lemma 6, with the initial matrices $X_{1}^{1}=$ $A_{1}^{T} Y B_{1}^{T}+C_{1}^{T} Z D_{1}^{T}, X_{2}^{1}=A_{2}^{T} Y B_{2}^{T}+C_{2}^{T} Z D_{2}^{T}$, where $Y \in R^{p \times q}$,
$Z \in R^{s \times t}$ are arbitrary, or, especially, $X_{1}^{1}=0$ and $X_{2}^{1}=0$, then the solution pair $X_{1}^{k}, X_{2}^{k}$ generated by Algorithm 3 is the least Frobenius norm solution of the matrix equations (1).

Suppose that Problem 1 is consistent. Obviously the solution set $S$ of (1) is nonempty. For given matrices pair $\widetilde{X}_{1} \in R^{k \times r}, \widetilde{X}_{2} \in R^{m \times n}$, we can write

$$
\begin{aligned}
& A_{1} X_{1} B_{1}+A_{2} X_{2} B_{2}=E, \\
& C_{1} X_{1} D_{1}+C_{2} X_{2} D_{2}=F, \\
& \Longleftrightarrow\left\{\begin{array}{c}
A_{1}\left(X_{1}-\widetilde{X}_{1}\right) B_{1}+A_{2}\left(X_{2}-\widetilde{X}_{2}\right) B_{2} \\
=E-A_{1} \widetilde{X}_{1} B_{1}-A_{2} \widetilde{X}_{2} B_{2}, \\
C_{1}\left(X_{1}-\widetilde{X}_{1}\right) D_{1}+C_{2}\left(X_{2}-\widetilde{X}_{2}\right) D_{2} \\
=F-C_{1} \widetilde{X}_{1} D_{1}-C_{2} \widetilde{X}_{2} D_{2} .
\end{array}\right.
\end{aligned}
$$

Let $\bar{X}_{1}=X_{1}-\widetilde{X}_{1}, \bar{X}_{2}=X_{2}-\widetilde{X}_{2}, \bar{E}=E-A_{1} \widetilde{X}_{1} B_{1}-A_{2} \widetilde{X}_{2} B_{2}$, and $\bar{F}=F-C_{1} \widetilde{X}_{1} D_{1}-C_{2} \widetilde{X}_{2} D_{2}$. Then Problem 2 is equivalent to find the least Frobenius norm solution pair of the system

$$
\begin{aligned}
& A_{1} \bar{X}_{1} B_{1}+A_{2} \bar{X}_{2} B_{2}=\bar{E}, \\
& C_{1} \bar{X}_{1} D_{1}+C_{2} \bar{X}_{2} D_{2}=\bar{F},
\end{aligned}
$$

which can be obtained using Algorithm 3 with the initial matrix pair $\bar{X}_{1}^{1}=A_{1}^{T} Y B_{1}^{T}+C_{1}^{T} Z D_{1}^{T}, \bar{X}_{2}^{1}=A_{2}^{T} Y B_{2}^{T}+C_{2}^{T} Z D_{2}^{T}$ where $Y \in R^{p \times q}$ and $Z \in R^{s \times t}$ are arbitrary, or especially, $\bar{X}_{1}^{1}=0, \bar{X}_{2}^{1}=0$, and the solution of the matrix optimal approximation Problem 2 can be represented as $\widehat{X}_{1}=\bar{X}_{1}^{k}+\widetilde{X}_{1}$, $\widehat{X}_{2}=\bar{X}_{2}^{k}+\widetilde{X}_{2}$.

\section{An Example}

In this section, we show a numerical example to illustrate the efficiency of Algorithm 3. All computations are performed by MATLAB 7. For the influence of the error of calculation, we consider the matrix $R$ as a zero matrix if $\|R\|<10^{-10}$.

Example 1. Consider the solution of the linear matrix equations

$$
\begin{aligned}
& A_{1} X_{1} B_{1}+A_{2} X_{2} B_{2}=E, \\
& C_{1} X_{1} D_{1}+C_{2} X_{2} D_{2}=F,
\end{aligned}
$$

where

$$
\begin{aligned}
& A_{1}=\left(\begin{array}{ccc}
139 & 105 & 54 \\
124 & 176 & 50 \\
159 & 35 & 175 \\
191 & 196 & 147
\end{array}\right) \\
& B_{1}=\left(\begin{array}{ccccc}
13 & 117 & 103 & 87 & 116 \\
198 & 85 & 67 & 45 & 152
\end{array}\right) \text {, } \\
& A_{2}=\left(\begin{array}{cc}
27 & 60 \\
2 & 132 \\
179 & 57 \\
40 & 94
\end{array}\right),
\end{aligned}
$$




$$
\begin{aligned}
& B_{2}=\left(\begin{array}{ccccc}
106 & 76 & 92 & 12 & 83 \\
128 & 157 & 114 & 121 & 61 \\
42 & 136 & 159 & 10 & 175
\end{array}\right), \\
& C_{1}=\left(\begin{array}{ccc}
3 & 88 & 192 \\
154 & 100 & 145 \\
194 & 43 & 82 \\
198 & 129 & 149 \\
158 & 64 & 54
\end{array}\right) \\
& D_{1}=\left(\begin{array}{ccc}
88 & 31 & 140 \\
71 & 135 & 146
\end{array}\right) \text {, } \\
& C_{2}=\left(\begin{array}{cc}
4 & 63 \\
115 & 3 \\
90 & 77 \\
9 & 137 \\
5 & 19
\end{array}\right) \\
& D_{2}=\left(\begin{array}{ccc}
96 & 90 & 55 \\
111 & 143 & 51 \\
24 & 179 & 173
\end{array}\right) \text {, } \\
& E=\left(\begin{array}{ccccc}
8433077 & 7598166 & 7137224 & 3600960 & 9942247 \\
11267058 & 9622068 & 9126164 & 4374104 & 12443246 \\
14871294 & 16123511 & 15980470 & 6538271 & 20550138 \\
16234322 & 14317981 & 13168541 & 7214805 & 18956522
\end{array}\right) \text {, } \\
& F=\left(\begin{array}{ccc}
8104691 & 10054438 & 13979223 \\
8672308 & 12546367 & 16384832 \\
7666584 & 11382258 & 13330665 \\
10613517 & 13831240 & 17573398 \\
4111503 & 5032847 & 7285952
\end{array}\right) .
\end{aligned}
$$

In this example, the numbers of the parameter matrices $A_{1}, A_{2}, B_{1}, B_{2}, C_{1}, C_{2}, D_{1}, D_{2}$ are larger than the numbers of the parameter matrices in the example of [32]. It can be verified that these matrix equations are consistent and have the solution as

$$
X_{1}=\left(\begin{array}{cc}
53 & 48 \\
32 & 129 \\
175 & 193
\end{array}\right), \quad X_{2}=\left(\begin{array}{ccc}
133 & 2 & 164 \\
174 & 27 & 86
\end{array}\right) .
$$

Let

$$
X_{1}^{0}=\left(\begin{array}{ll}
0 & 0 \\
0 & 0 \\
0 & 0
\end{array}\right), \quad X_{2}^{0}=\left(\begin{array}{lll}
0 & 0 & 0 \\
0 & 0 & 0
\end{array}\right) .
$$

(1) Using Algorithm 3 and iterate 10309 steps, we obtain the least Frobenius norm solution pair of the matrix equation in Example 1 as follows:

$$
\begin{gathered}
X_{1}=\left(\begin{array}{cc}
53.0000 & 48.0000 \\
32.0000 & 129.0000 \\
175.0000 & 193.0000
\end{array}\right), \\
X_{2}=\left(\begin{array}{ccc}
133.0000 & 2.0000 & 164.0000 \\
174.0000 & 27.0000 & 86.0000
\end{array}\right) .
\end{gathered}
$$

The obtained sequence $\|\Delta X\|$ are presented in Figure 1.

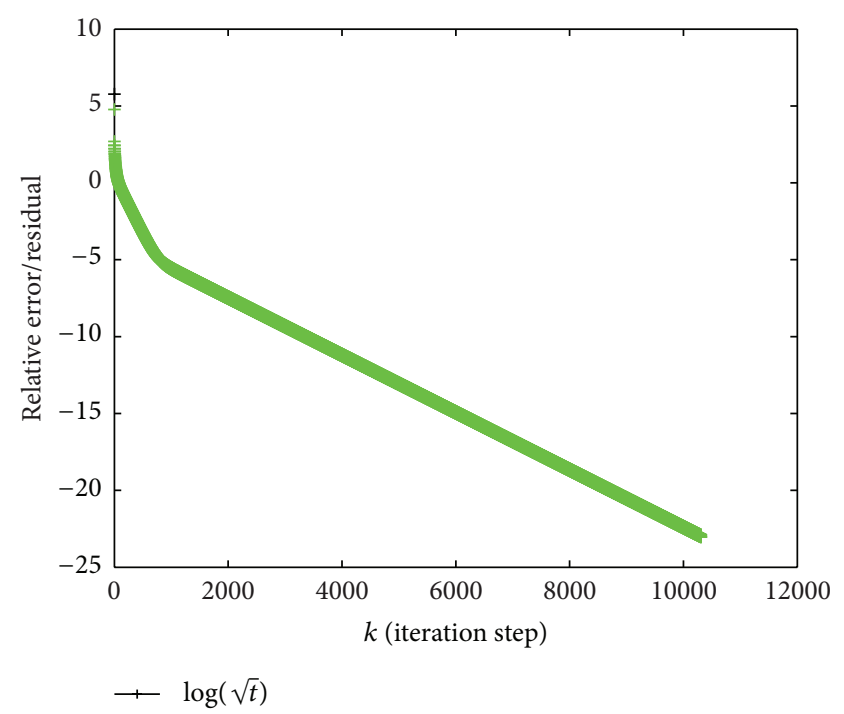

FIgure 1: The obtained sequence $\|\Delta X\|$ by Algorithm 3 for Example 1.

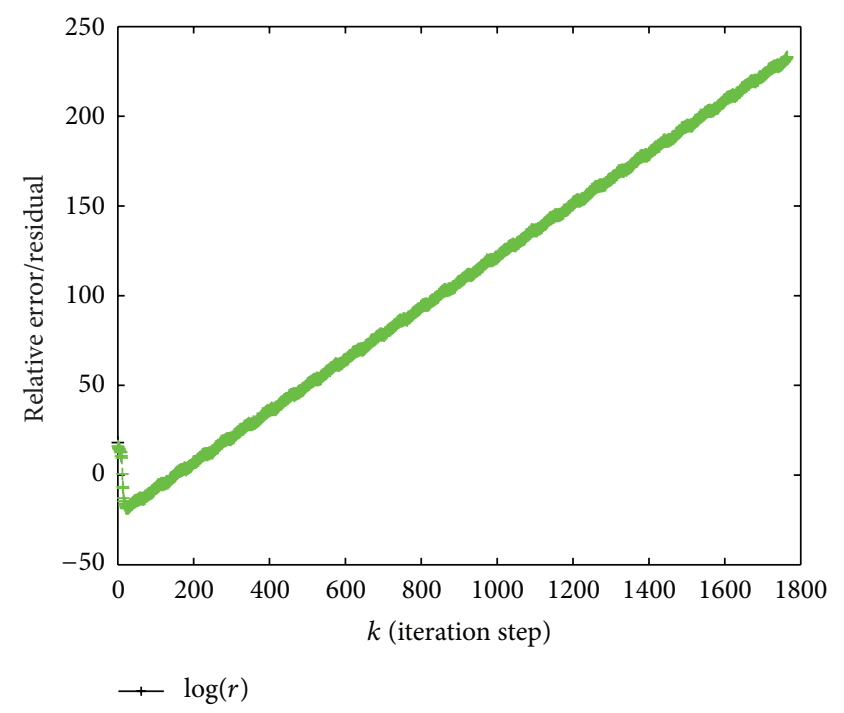

FIGURE 2: The relative error of the solution and the residual by the algorithm of [32] for Example 1.

(2) Using the algorithm of [32], to this example, the iteration is not convergent. The obtained result is presented in Figure 2.

This numerical example demonstrates that our algorithm has merits of good numerical stability and ease to program.

\section{Acknowledgments}

This research was supported by the Grants from the Key Project of Scientific Research Innovation Foundation of Shanghai Municipal Education Commission (13ZZ080), the National Natural Science Foundation of China (11171205), the Natural Science Foundation of Shanghai (11ZR1412500), 
and the Nature Science Foundation of Anhui Provincial Education (ky2008b253, KJ2013A248).

\section{References}

[1] T. Meng, "Experimental design and decision support, in expert systems," in The Technology of Knowledge Management and Decision Making for the 21st Century, C. Leondes, Ed., vol. 1, Academic Press, New York, NY, USA, 2001.

[2] M. Dehghan and M. Hajarian, "An iterative algorithm for solving a pair of matrix equations $A Y B=E, C Y D=F$ over generalized centro-symmetric matrices," Computers \& Mathematics with Applications, vol. 56, no. 12, pp. 3246-3260, 2008.

[3] M. Dehghan and M. Hajarian, "An iterative algorithm for the reflexive solutions of the generalized coupled Sylvester matrix equations and its optimal approximation," Applied Mathematics and Computation, vol. 202, no. 2, pp. 571-588, 2008.

[4] A. L. Andrew, "Solution of equations involving centrosymmetric matrices," Technometrics, vol. 15, no. 2, pp. 405-407, 1973.

[5] A. Navarra, P. L. Odell, and D. M. Young, "A representation of the general common solution to the matrix equations $A_{1} X B_{1}=C_{1}$ and $A_{2} X B_{2}=C_{2}$ with applications," Computers \& Mathematics with Applications, vol. 41, no. 7-8, pp. 929-935, 2001.

[6] Y.-X. Peng, X.-Y. Hu, and L. Zhang, "An iterative method for symmetric solutions and optimal approximation solution of the system of matrix equations $A_{1} X B_{1}=C_{1}, A_{2} X B_{2}=C_{2}$," Applied Mathematics and Computation, vol. 183, no. 2, pp. 1127-1137, 2006.

[7] Z.-H. Peng, X.-Y. Hu, and L. Zhang, "An efficient algorithm for the least-squares reflexive solution of the matrix equation $A_{1} X B_{1}=C_{1} ; A_{2} X B_{2}=C_{2}$," Applied Mathematics and Computation, vol. 181, no. 2, pp. 988-999, 2006.

[8] X. Sheng and G. Chen, "A finite iterative method for solving a pair of linear matrix equations $(A X B, C X D)=(E, F)$," Applied Mathematics and Computation, vol. 189, no. 2, pp. 1350-1358, 2007.

[9] N. Li and Q. W. Wang, "Iterative algorithm for solving a class of quaternion matrix equation over the generalized $(P, Q)$ reflexive matrices," Abstract and Applied Analysis, vol. 2013, Article ID 831656, 15 pages, 2013.

[10] A.-G. Wu, G. Feng, G.-R. Duan, and W.-J. Wu, "Finite iterative solutions to a class of complex matrix equations with conjugate and transpose of the unknowns," Mathematical and Computer Modelling, vol. 52, no. 9-10, pp. 1463-1478, 2010.

[11] A.-G. Wu, G. Feng, G.-R. Duan, and W.-J. Wu, "Iterative solutions to coupled Sylvester-conjugate matrix equations," Computers \& Mathematics with Applications, vol. 60, no. 1, pp. 54-66, 2010.

[12] A.-G. Wu, B. Li, Y. Zhang, and G.-R. Duan, "Finite iterative solutions to coupled Sylvester-conjugate matrix equations," Applied Mathematical Modelling, vol. 35, no. 3, pp. 1065-1080, 2011.

[13] Y.-X. Yuan, "On the minimum norm solution of matrix equation $A X B=E ; C X D=F$," Journal of East China Shipbuilding Institute, vol. 15, no. 3, pp. 34-37, 2001.

[14] S. K. Mitra, "Common solutions to a pair of linear matrix equations $A_{1} X B_{1}=C_{1}$ and $A_{2} X B_{2}=C_{2}$, vol. 74, pp. 213-216, 1973.
[15] N. Shinozaki and M. Sibuya, "Consistency of a pair of matrix equations with an application," Keio Science and Technology Reports, vol. 27, no. 10, pp. 141-146, 1975.

[16] J. W. van der Woude, Freeback decoupling and stabilization for linear systems with multiple exogenous variables [Ph.D. thesis], Technical University of Eindhoven, Eindhoven, The Netherlands, 1987.

[17] M. Dehghan and M. Hajarian, "The general coupled matrix equations over generalized bisymmetric matrices," Linear Algebra and Its Applications, vol. 432, no. 6, pp. 1531-1552, 2010.

[18] M. Dehghan and M. Hajarian, "The reflexive and anti-reflexive solutions of a linear matrix equation and systems of matrix equations," The Rocky Mountain Journal of Mathematics, vol. 40, no. 3, pp. 825-848, 2010.

[19] Q.-W. Wang, J.-H. Sun, and S.-Z. Li, "Consistency for bi(skew)symmetric solutions to systems of generalized Sylvester equations over a finite central algebra," Linear Algebra and Its Applications, vol. 353, pp. 169-182, 2002.

[20] Q.-W. Wang, "Bisymmetric and centrosymmetric solutions to systems of real quaternion matrix equations," Computers \& Mathematics with Applications, vol. 49, no. 5-6, pp. 641-650, 2005.

[21] Q.-W. Wang, "A system of matrix equations and a linear matrix equation over arbitrary regular rings with identity," Linear Algebra and Its Applications, vol. 384, pp. 43-54, 2004.

[22] F. Ding, P. X. Liu, and J. Ding, "Iterative solutions of the generalized Sylvester matrix equations by using the hierarchical identification principle," Applied Mathematics and Computation, vol. 197, no. 1, pp. 41-50, 2008.

[23] J. Ding, Y. Liu, and F. Ding, "Iterative solutions to matrix equations of the form $A_{i} X B_{i}=F_{i}$," Computers \& Mathematics with Applications, vol. 59, no. 11, pp. 3500-3507, 2010.

[24] L. Xie, J. Ding, and F. Ding, "Gradient based iterative solutions for general linear matrix equations," Computers \& Mathematics with Applications, vol. 58, no. 7, pp. 1441-1448, 2009.

[25] L. Xie, Y. Liu, and H. Yang, "Gradient based and least squares based iterative algorithms for matrix equations $A X B+C X^{T} D=$ F," Applied Mathematics and Computation, vol. 217, no. 5, pp. 2191-2199, 2010.

[26] Z.-Y. Li, Y. Wang, B. Zhou, and G.-R. Duan, "Least squares solution with the minimum-norm to general matrix equations via iteration," Applied Mathematics and Computation, vol. 215, no. 10, pp. 3547-3562, 2010.

[27] Z.-Y. Li, B. Zhou, Y. Wang, and G.-R. Duan, "Numerical solution to linear matrix equation by finite steps iteration," IET Control Theory \& Applications, vol. 4, no. 7, pp. 1245-1253, 2010.

[28] B. Zhou, J. Lam, and G.-R. Duan, "On Smith-type iterative algorithms for the Stein matrix equation," Applied Mathematics Letters, vol. 22, no. 7, pp. 1038-1044, 2009.

[29] B. Zhou, J. Lam, and G.-R. Duan, "Gradient-based maximal convergence rate iterative method for solving linear matrix equations," International Journal of Computer Mathematics, vol. 87, no. 1-3, pp. 515-527, 2010.

[30] Y.-B. Deng, Z.-Z. Bai, and Y.-H. Gao, "Iterative orthogonal direction methods for Hermitian minimum norm solutions of two consistent matrix equations," Numerical Linear Algebra with Applications, vol. 13, no. 10, pp. 801-823, 2006.

[31] Y.-T. Li and W.-J. Wu, "Symmetric and skew-antisymmetric solutions to systems of real quaternion matrix equations," Computers \& Mathematics with Applications, vol. 55, no. 6, pp. 1142-1147, 2008. 
[32] M. Dehghan and M. Hajarian, "An efficient algorithm for solving general coupled matrix equations and its application," Mathematical and Computer Modelling, vol. 51, no. 9-10, pp. 1118-1134, 2010.

[33] M. Dehghan and M. Hajarian, "On the reflexive and antireflexive solutions of the generalised coupled Sylvester matrix equations," International Journal of Systems Science, vol. 41, no. 6, pp. 607-625, 2010.

[34] B. Zhou, Z.-Y. Li, G.-R. Duan, and Y. Wang, "Weighted least squares solutions to general coupled Sylvester matrix equations," Journal of Computational and Applied Mathematics, vol. 224, no. 2, pp. 759-776, 2009.

[35] I. Jonsson and B. Kågström, "Recursive blocked algorithm for solving triangular systems. I. One-sided and coupled Sylvestertype matrix equations," ACM Transactions on Mathematical Software, vol. 28, no. 4, pp. 392-415, 2002.

[36] B. Zhou, G.-R. Duan, and Z.-Y. Li, "Gradient based iterative algorithm for solving coupled matrix equations," Systems \& Control Letters, vol. 58, no. 5, pp. 327-333, 2009.

[37] I. Jonsson and B. Kågström, "Recursive blocked algorithm for solving triangular systems. II. Two-sided and generalized Sylvester and Lyapunov matrix equations," ACM Transactions on Mathematical Software, vol. 28, no. 4, pp. 416-435, 2002.

[38] A.-P. Liao and Y. Lei, "Least-squares solution with the minimum-norm for the matrix equation $(A X B, G X H)=$ $(C, D)$," Computers \& Mathematics with Applications, vol. 50, no. 3-4, pp. 539-549, 2005.

[39] J. Cai and G. Chen, "An iterative algorithm for the least squares bisymmetric solutions of the matrix equations $A_{1} X B_{1}=$ $C_{1}, A_{2} X B_{2}=C_{2}$," Mathematical and Computer Modelling, vol. 50, no. 7-8, pp. 1237-1244, 2009.

[40] F. Yin and G.-X. Huang, "An iterative algorithm for the least squares generalized reflexive solutions of the matrix equations $A X B=E ; C X D=F, "$ Abstract and Applied Analysis, vol. 2012, Article ID 857284, 18 pages, 2012.

[41] Y.-X. Peng, X.-Y. Hu, and L. Zhang, "An iteration method for the symmetric solutions and the optimal approximation solution of the matrix equation $A X B=C$, Applied Mathematics and Computation, vol. 160, no. 3, pp. 763-777, 2005. 


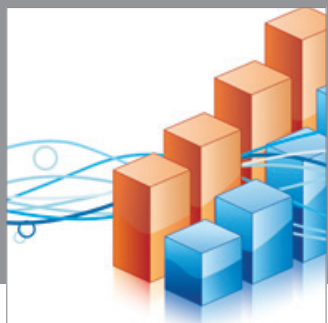

Advances in

Operations Research

mansans

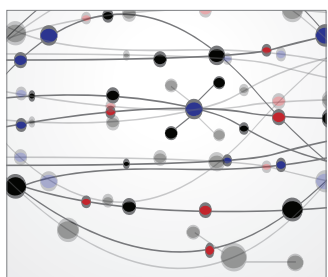

The Scientific World Journal
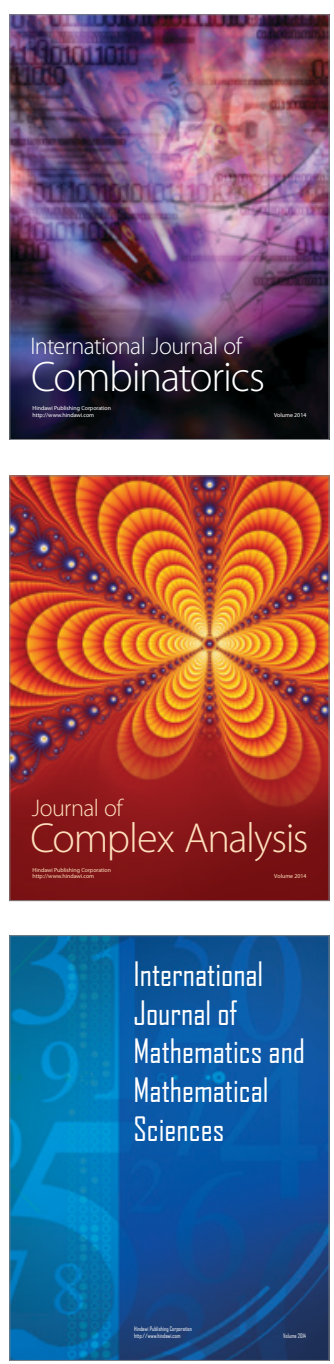
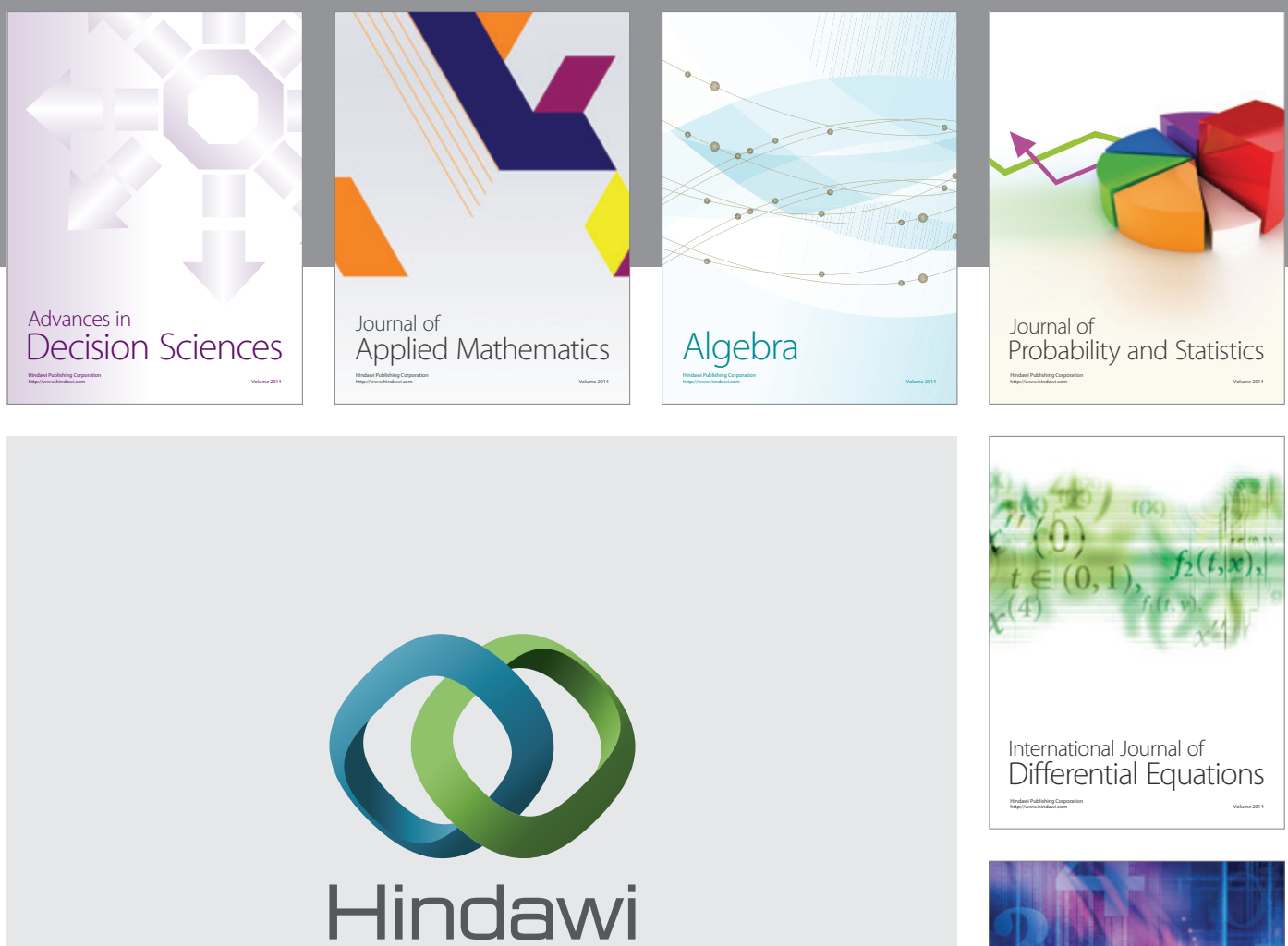

Submit your manuscripts at http://www.hindawi.com
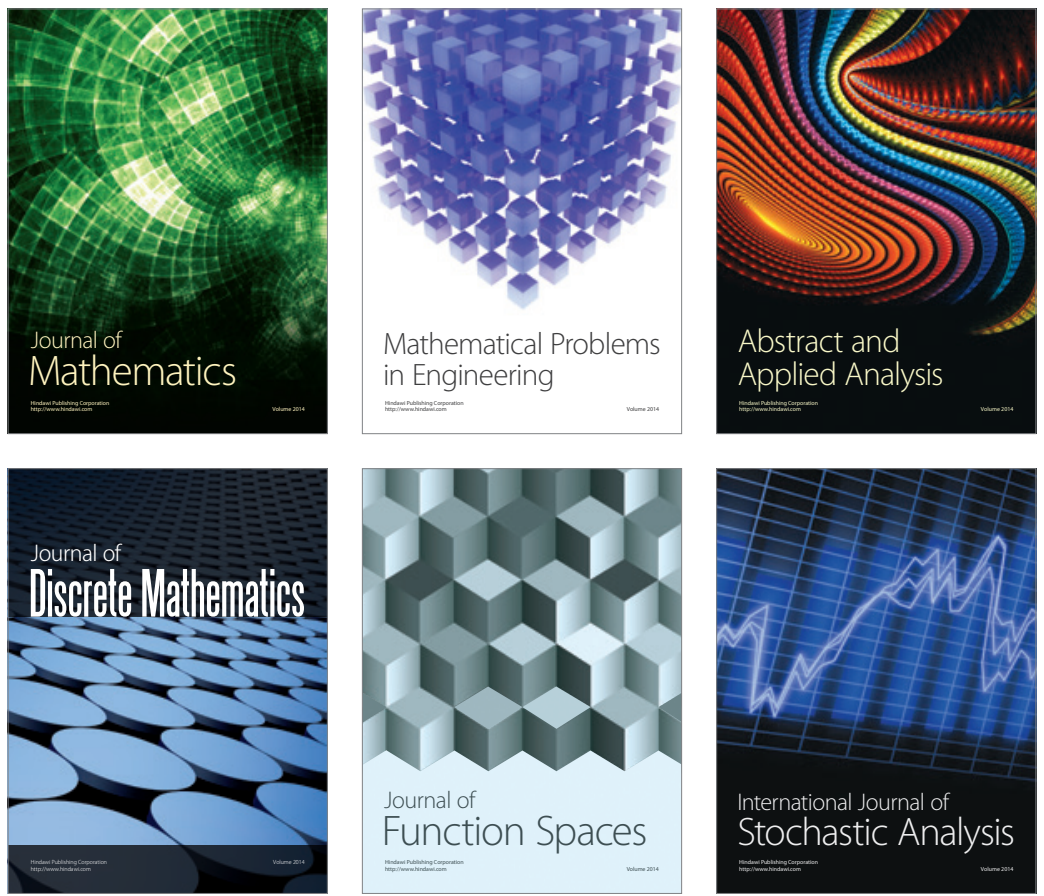

Journal of

Function Spaces

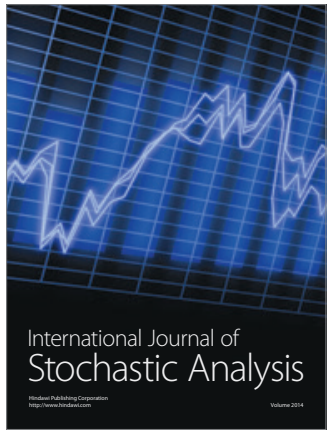

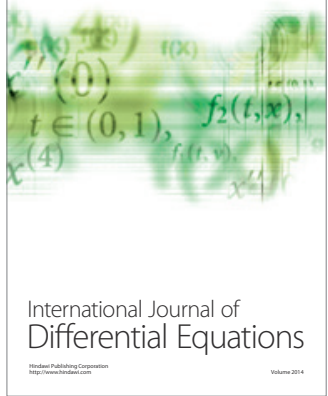
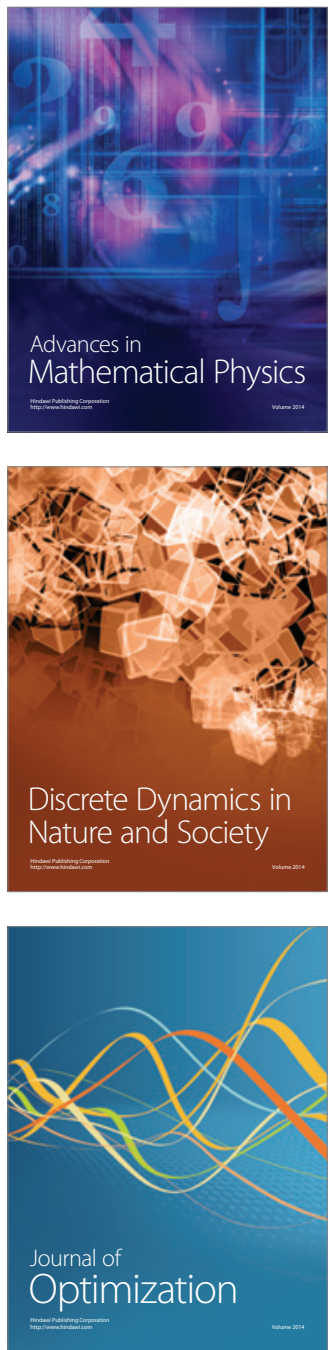